\title{
Magnetic Shape Memory Alloys as smart materials for micro-positioning devices
}

\author{
A. Hubert ${ }^{1 *}$, N. Calchand ${ }^{1}$, Y. Le Gorrec ${ }^{1}$, J.-Y. Gauthier ${ }^{2}$ \\ ${ }^{1}$ Institut Femto-ST UMR 6174, UFC/ENSMM/UTBM/CNRS, \\ 24 rue Alain Savary, 25000 Besançon, France \\ ${ }^{2}$ Laboratoire Ampère UMR 5005, Université de Lyon/CNRS, \\ INSA Lyon, bâtiment A. de Saint-Exupéry, Avenue Jean Capelle, \\ 69621 Villeurbanne cedex, France \\ *corresponding author, E-mail: arnaud. hubert@femto-st. fr
}

\begin{abstract}
In the field of microrobotics, actuators based on smart materials are predominant because of very good precision, integration capabilities and high compactness. This paper presents the main characteristics of Magnetic Shape Memory Alloys as new candidates for the design of micromechatronic devices. The thermo-magneto-mechanical energy conversion process is first presented followed by the adequate modeling procedure required to design actuators. Finally, some actuators prototypes realized at the Femto-ST institute are presented, including a push-pull bidirectional actuator. Some results on the control and performances of these devices conclude the paper.
\end{abstract}

\section{Introduction}

Electromagnetic devices have become promising contenders in the area of ultra-high precision manufacturing, manipulation and sensing. Most of these promising new technologies uses active/adaptive materials as a base for actuation principles because of compactness, integrations and smart use capabilities [1,2]. This paper reports recent results obtained using a new type of smart material called Magnetic Shape Memory Alloy (MSMA) which is an alloy of Ni-Mn-Ga.

Currently, most of the high-precision devices use piezoelectric materials such as PZT because of their highdynamic bandwidth and their nearly linear behaviour. Piezoelectric materials are used to design micropositioning devices by using classical mode of working $[3,4]$ or by using stick and slip mode of working $[5,6]$. In the field of magnetic active materials, the main applications use magnetostrictive materials $[7,8]$ especially TerfenolD. This material is the most used because of the important progress in its performances since its first use in 1950 by the Naval Ordnance Laboratory in the USA and its high magneto-mechanical coupling compared to the other magnetostrictive materials. Examples of actuators and positioning devices using magnetostrictive materials can be found in $[9,10,11]$.

Piezoelectric and magnetostrictive materials are the most used adaptive materials for positioning application. Nevertheless, alternative solutions exist and MSMAs are a class of active materials which typically generate $6 \%$ strain in response to externally applied magnetic fields [12]. In contrast to conventional heat driven shape memory alloys (SMAs), the magnetically driven MSMAs exhibit higher operating frequency, making them attractive for actuation applications. Most of the work on characterization and modeling of MSMAs has been focused on quasistatic actuation, i.e., strain dependence on magnetic field. Very few studies take into account the dynamical behaviour and even fewer propose MSMA based actuator prototypes. The purpose of this paper is to present a modeling procedure taking into account the complex magneto-mechanical behaviors of MSMA and the dynamical characteristics of actuators at the same time, to design new micropositioning devices.

Among all the offer concerning magnetic active materials, Magnetic Shape Memory Alloy is a relatively new candidate. It was first experimented in 1995/1996 at the MIT for the Ni-Mn-Ga alloy [13] and at the University of Minnesota for the Fe-Pd alloy [14]. Since these first works, lots of researchs have been conducted all around the world (see $[15,16]$ for reviews) but very few concrete applications exist today due to the complex dynamic behaviour of MSMA and due to the difficulties to improve its manufacturing process. Currently, only single crystal samples present significant magneto-mechanical coupling characteristics and even if a lot of studies are conducted concerning thin films deposition and polycrystal samples [17, 18], these types of structures are presently less adequate for actuation applications because of a lower magneto-mechanical coupling.

MSMA can be seen as a mixture of a classical shape memory alloy (SMA) and a magnetostrictive material. In a way, its microscopic behaviour is similar to SMAs but strain is not only due to a martensite/austenite phase transformation but is also due to a martensite reorientation under magnetic fields. In this second mode of working martensite rearrangement-, MSMA can be compared with standard magnetostrictive materials but with a much more important magneto-mechanical coupling ( $6 \%$ of maximal strain for Ni-Mn-Ga instead of $0.16 \%$ for Terfenol-D). 
This paper first explains the working principle of this active material and gives some tools for static and dynamic behaviour modeling. Then, some design rules will be presented to propose efficient actuators. These modeling works will be used as guidelines to design a new micro-positioning stage. It will be based on two antagonist MSMA-based actuators to perform a one-degree-offreedom linear axis stage with a full-range motion of the order of millimeters and a micrometers precision. Measurements conducted on the prototype will be presented and commented.

\section{Basis on MSMA modeling and behaviour}

\subsection{MSMA Properties and Characteristics}

As seen before, the working principle of MSMA is quite similar to the working of SMA but its actuation can also result from magnetic field application. This second mode of working allows to increase significantly the dynamical bandwidth of the device because it uses a magnetomechanical energy conversion process instead of a thermomechanical. Since fifteen years, these materials knew some improvements concerning mainly the working temperature range and the maximum available strain. When actuated by magnetic fields, a large strain (6 to $10 \%)$ is obtained with a response-time in the range of milliseconds (to compare with tenth of seconds or even seconds for SMA). The MSMA used in this paper is a non-stoechiometric NiMn-Ga monocrystal corresponding to the most currently used MSMA materials. In this alloy, the martensite phase appears in three different variants corresponding to the three possible crystallographic directions in the sample (see Fig. 1 (a)).

The martensitic reorientation principle is presented in Fig. 1 (b): at high temperature, the MSMA sample is in austenitic phase (A). After a cooling process, the austenite phase is transformed into a martensite phase without any favoured variants. Therefore the resulting sample contains martensite variants into three equal portions (M1, M2 and M3). If a mechanical stress is applied in a specific direction, then the fraction of variant with its short axis in this direction grows. If this stress is high enough then the sample will only contain this variant (for example M2 in Fig. 1 (b)). If the stress decreases, the volume fraction of the M2 variant also decrease but in an irreversible behaviour (large hysteresis). In a same way, if a magnetic field is applied, the variant with its easy magnetization axis in the field direction, is favoured. For MSMA, the easy magnetization direction is the same as the short axis of the M1 variant. In this case, this variant fraction increases as shown in Fig. 1 (b) by the application of a magnetic field perpendicular to the stress field. The balance between magnetic field and mechanical stress allows a control of the macroscopic strain. With a pre-stress, one can therefore obtain an actuator driven by the magnetic field only. Austenite phase can also be recovered by heating.

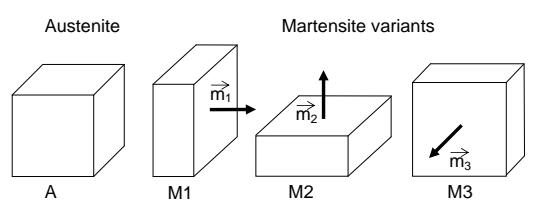

(a)

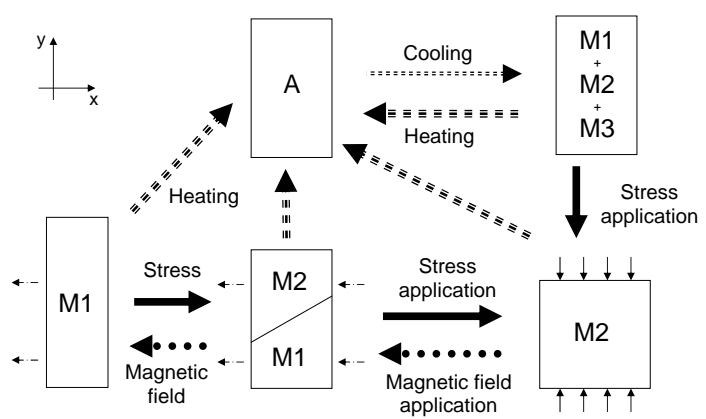

(b)

Figure 1: MSMA behaviour: (a) austenite phase and the three martensite variants and (b) martensitic reorientation: effects of mechanical stress, magnetic field and temperature.

Besides these interesting properties, the material also has some important drawbacks. They are the brittleness of the single-crystal, the high required magnetic field (400 $\mathrm{kA} / \mathrm{m}$ ), the large dependence of the material parameters on temperature changes, a small blocking stress (2-3 MPa) and a large hysteretical thermo-magneto-mechanical behaviour. More details about the structural properties of this material can be found in [15].

\subsection{Quasi-Static Modeling of MSMA}

Two theories can be used to model the behaviour of MSMA:

- The first one is based on microscopic properties and physics of solid. The following papers are representative of the works done on MSMA materials with this approach $[19,20,21,22]$.

- The second one is based on macroscopic properties and thermodynamics. In $[23,24,25]$, such models are proposed whereas in $[26,27,28]$, a more mechatronics-based approach is taken. They explicitly take into account the salient features (dynamics of system) required to make actuators.

While the first theory is prefered by physicists, the second one is prefered by engineers and is best-suited to design actuators and micropositioning applications. This paper will focus mainly on actuator design and only few details will be given concerning our modelling works (recent results could 
be found in [29]). To simplify the mathematical expressions, only isothermal actuation in the $x y$ plan is considered in this paper with a control of position along the $x$ axis. In such a configuration, only M1 and M2 variants are relevant.

To model quasi-static behaviour of MSMA using the thermodynamic approach, it is first necessary to propose an expression for the internal energy $\mathcal{U}$ or a similar thermodynamic potentiel using Legendre Transform (for exemple, Gibbs free energy $\mathcal{G}$ or Helmholtz free energy $\mathcal{F}$ ). This potential must take into account all physical phenomena that are necessary to capture the experimental behaviour [30] namely:

- chemical energy,

- thermal energy,

- mechanical energy,

- magnetic energy.

In the case of MSMA, an irreversible behaviour takes place when phase rearrangement or transformation appears. This one is associated with a dissipation function and a Clausius-Duhem inequality is able to take into account the irreversibility and the increase of the total entropy of the system. In our work, thermodynamics of irreversible processes with internal variables is used. These internal variables are quantities used as additionnal internal state variables. This additional knowledge from the micro-physical material behaviour permits to compute explicitely the available work in the case of irreversible processes.

The complete thermodynamic expressions of MSMA used by authors are fully described in [28] and the internal variables are linked to a micro/macro approach. The Fig. 2 displays a Representative Elementary Volume of the MSMA, i.e. a mean - macroscopic - representation of the microscopic structure of this material. This one allows to express different internal variables:

- $z_{o}$ : austenite volume fraction,

- $z_{k}$ : volume fraction of martensite variant $k$ ( $k \in$ $\{1 ; n\})$, i.e. the martensite presents $n$ different variants. Let $\sum_{k=1}^{n} z_{k}=\left(1-z_{o}\right)$ be the global fraction of martensite,

- $\alpha$ and $(1-\alpha)$ the proportions of the Weiss domains inside a variant representing the Representative Elementary Volume,

- $\theta$ the rotation angle of the magnetization vector associated to the two Weiss domains of variant $M_{2}$. Indeed, under the magnetic field $\vec{H}$, this magnetization rotates in order to become parallel to the magnetic field. As the field $\vec{H}$ is parallel to $\vec{x}$, there is no rotation of Weiss domains of variant $M_{1}$.

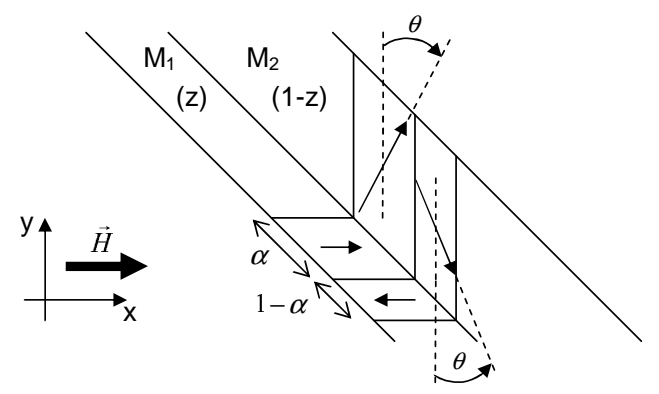

Figure 2: Representative Elementary Volume when the MSM sample is only composed of two martensite variants $M_{1}$ and $M_{2}\left(z=z_{1}\right.$ and $\left.1-z=z_{2}\right)$.

In [28], we proposed the following expression for the Gibbs free energy :

$$
\begin{aligned}
& \rho G(\sigma, H, T, z, \alpha, \theta)=C_{p}\left[\left(T-T_{o}\right)-T \cdot \log \frac{T}{T_{o}}\right] \\
& -\sigma \gamma z-\frac{\sigma^{2}}{2 E}+K_{12} z(1-z) \\
& -\mu_{o} M_{S}\left(z\left((2 \alpha-1) H-\frac{M_{S}}{2 \chi_{a}}(2 \alpha-1)^{2}\right)\right. \\
& \left.+(1-z)\left((\sin \theta) H-\frac{M_{S}}{2 \chi_{t}}(\sin \theta)^{2}\right)\right)
\end{aligned}
$$

where $\sigma, H$ and $T$ are the stress, the applied magnetic field and the temperature of the MSMA sample, respectively. All other variables are material parameters and can be experimentally identified (see [31]). In this expression, the magneto-thermo-mechanical coupling is expressed by the choice of the internal variable $z$. The total strain $\varepsilon$ can therefore be deduced as:

$$
\varepsilon=-\frac{\partial(\rho G)}{\partial \sigma}=\frac{\sigma}{E}+\gamma z=\varepsilon^{e}+\varepsilon^{d t w}
$$

with $\varepsilon^{e}$ the elastic strain and $\varepsilon^{d t w}$ the strain resulting from a rearrangement between M1 and M2 variants $(\gamma=0.06$ is the maximal strain due to a complete martensitic rearrangement). The magnetization $M$ can also be computed as:

$$
\begin{aligned}
\mu_{o} M & =-\frac{\partial(\rho G)}{\partial H} \\
& =\mu_{o} M_{S}((2 \alpha-1) z+\sin \theta(1-z))
\end{aligned}
$$

With this modelling procedure, a thermodynamical force $\pi^{z}$ associated with the $z$ fraction of martensite is also deduced as:

$$
\begin{aligned}
& \pi^{z}=-\frac{\partial \rho G}{\partial z}=\sigma \gamma-K_{12}(1-2 z) \\
& +\mu_{o} M_{S}\left[(2 \alpha-1) H-\frac{M_{S}}{2 \chi_{a}}(2 \alpha-1)^{2}\right. \\
& \left.-H \sin \theta+\frac{M_{S}}{2 \chi_{t}} \sin ^{2} \theta\right]
\end{aligned}
$$

This term can be used explicitly in the Clausius-Duhem inequality to compute the dissipation $d D$, i.e. the irreversibility of the processes, associated with a phase rearrangement 7 
processes.

$$
d D=\pi^{z} d z \geq 0
$$

This explicit expression can therefore be used to integrate the available work $\int d \mathcal{W}$ resulting from the energy conversion process. This quasistatic modeling based on thermodynamics of irreversible processes can be used to predict accuratelly the stress/strain behaviour of MSMA samples as a function of the applied magnetic field and applied external stress. A comparison between simulation and experimental results are plotted in Fig. 3 (see [28]).
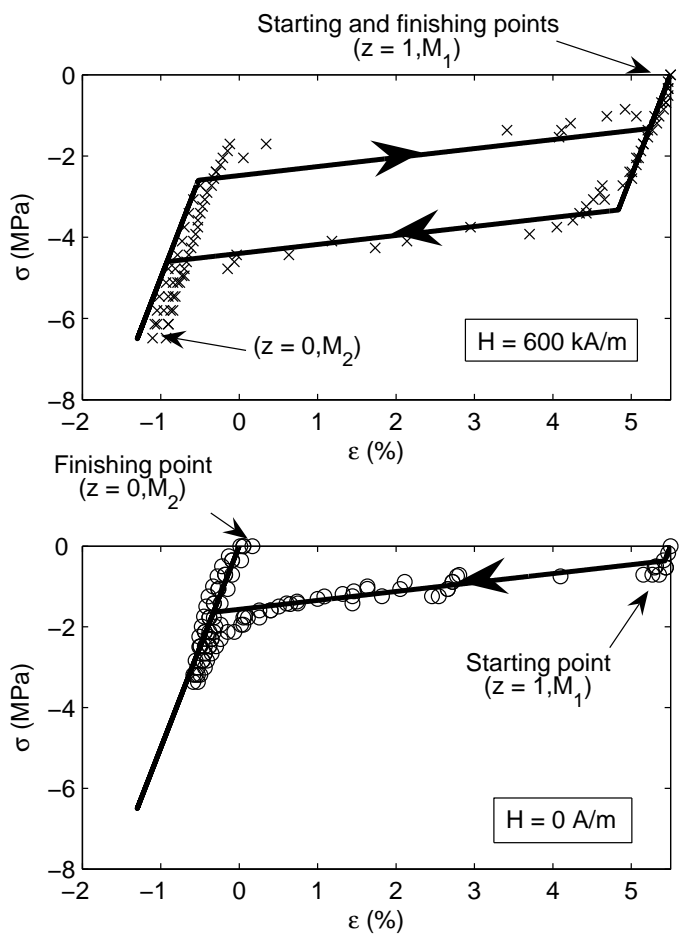

Figure 3: Strain vs stress plots for two different magnetic fields: model prediction (solid line) and experiments (crosses or circles) [28].

\subsection{Dynamic modeling of MSMA based actuators}

At this point, it should be emphasized that an actuator is not only a smart material but a more complex mechatronics device which includes a smart material, a mechanical structure, a power supply, a load, its associated control system and a few other components. To model the dynamic behaviour of such a device, the authors proposed in [31] to associate thermodynamics of irreversible processes with an Hamiltonian modeling. The irreversible behaviour associated with the MSMA non-linearity can be captured with internal variables and the behaviour of other components can be capture with generalized coordinates $\mathbf{q}$ and generalized momenta $\mathbf{p}$. This association allows to extend the "classical" Hamiltonian formalism to the non-conservative case (open and dissipative) with geometric or kinematic constraints. The Hamiltonian formalism uses an energy func- tion - a thermodynamic potential - corresponding to the total energy of the system, the Hamiltonian function $\mathcal{H}(\mathbf{q}, \mathbf{p})$ $[32,33]$.

For a conservative system - a thermodynamically close and non-dissipative system - , we simply have: $\mathcal{H}(\mathbf{q}, \mathbf{p})=$ $\mathcal{T}(\mathbf{p})+\mathcal{V}(\mathbf{q})$ with $\mathcal{T}$ and $\mathcal{V}$ the kinetic and potential/internal energy of the system. But an open system as our actuator means that it exchanges some energy with external environment. This is the case, for example, when the system is controlled with an external generalized force $\mathbf{f}_{e x t}$ depending explicitly on the time $t$ (we then speak about nonautonomous systems). For our system, the external generalized force is the current or the voltage - depending on the type of control - supplied by the electronic power converter. Furthermore, a dissipative system means that some part of the inner energy is non-available after any motion of the system as claimed by irreversibility and the ClausiusDuhem inequality. This could be computed using dissipated energy during any motion. Finally, system with constraints means that there is some topologic constraints on the admissible motion of this system. These geometric or kinematic constraints can be taken into account in the dynamical equations using a Lagrange multiplier technique. To include all these phenomena, we make use of an extented Hamiltonian function $\mathcal{H}^{\prime}$ with these features:

- The external generalized forces $\mathbf{f}_{\text {ext }}(\mathbf{q}, t)$ are taking into account in the variation of $\mathcal{H}^{\prime}$ by adding the influence of their virtual works $\delta \mathcal{W}_{\text {ext }}=\mathbf{f}_{\text {ext }}(\mathbf{q}, t) \cdot \delta \mathbf{q}$.

- Dissipations by static and viscous frictions are taking into account by adding their dissipated energies variations $\delta \mathcal{Q}_{s}(\mathbf{q})$ and $\delta \mathcal{Q}_{v}(\dot{\mathbf{q}})$. The dissipation by viscous friction $\mathcal{Q}_{v}(\dot{\mathbf{q}})$ is computed with a Rayleigh dissipation function $\mathcal{R}(\dot{\mathbf{q}})$ like $\mathcal{Q}_{v}(\dot{\mathbf{q}})=\int_{t_{1}}^{t_{2}} \mathcal{R}(\dot{\mathbf{q}}) d t$.

- The holonomic kinematic constraints $\mathbf{c}(\mathbf{q})=0$ are taking into account with a Lagrange multipliers technique by adding the term $\lambda \cdot \delta \mathbf{c}(\mathbf{q})$ to the variation $\delta \mathcal{H}^{\prime}$.

Finally, we have:

$$
\delta \mathcal{H}^{\prime}=\delta \mathcal{H}-\mathbf{f}_{e x t} \cdot \delta \mathbf{q}-\delta \mathcal{Q}_{s}-\delta \mathcal{Q}_{v}+\lambda \cdot \delta \mathbf{c}
$$

The Hamilton principle using $\delta \mathcal{H}^{\prime}$ gives the following Hamilton equations:

$$
\left\{\begin{array}{l}
\dot{q}_{i}=\frac{\partial \mathcal{H}}{\partial p_{i}} \\
\dot{p}_{i}=-\frac{\partial \mathcal{H}}{\partial q_{i}}-\frac{\partial \mathcal{R}}{\partial \dot{q}_{i}}+\frac{\partial \mathcal{Q}_{s}}{\partial q_{i}}+f_{\text {ext }, i}-\lambda_{i} \cdot \frac{\partial c_{i}}{\partial q_{i}}
\end{array}\right.
$$

These equations were used to model the dynamic behaviour of a simple actuator using MSMA and described in [31] and in the Fig. 4 (a). The comparison between theoretical and experimental dynamical response for an open loop control (voltage step) are reported in the Fig. 4 (b) and shows that the predictions given by this modelling procedure are quite accurate (see [31] for more details). 

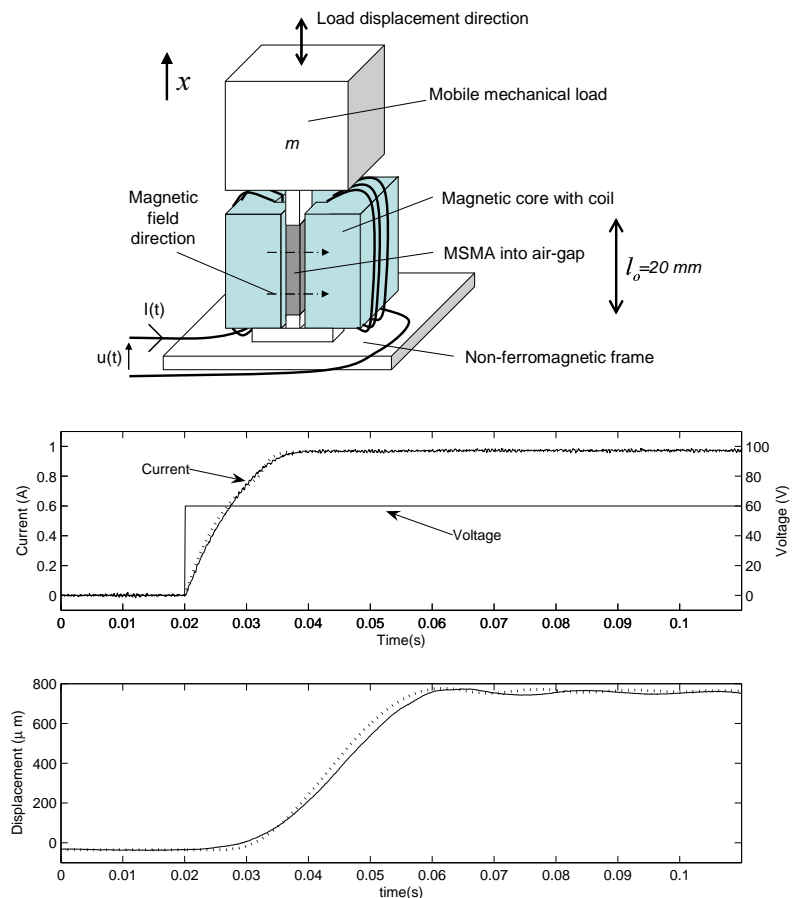

(b)

Figure 4: (a) description of a simple MSMA actuator, (b) dynamic behaviour of the system: voltage, current and displacement $v s$ time (simulation: dotted line and experimental results: solid line) [31].

\section{MSMA as an active material for actuator design}

\subsection{General design considerations}

Very few prototypes of actuators using MSMA are reported in the literature. The majority use the simple principle described previously in Fig. 4 (a), [34, 35, 36] and some few others use in addition the Inchworm or stick and slip principles $[37,38]$. The descriptions and models presented in authors previous papers and summed up in the previous sections give a better understanding of MSMA behaviour. This knowledge can therefore be used to design new actuators and to improve the performances of these devices. One of our latest version of actuators will be described in the following. Its characteristics and performances will be discussed.

\subsection{Push-Pull Configuration}

The study of the simple actuator described previously and in [31] shows that some advantages and disavantages of MSMA must be exploited to design competitive actuators compared to actuators using other active materials. First, it should be stressed that MSMA is not, at the moment, competitive for applications where other active materials are well-established. In particular for very high precision positioning (better than one micrometer) or for very small displacements (in the nanometer range), piezoelectric ma- terials are far more competitive. In addition, if very low bandwidth and high forces are required, thermal or classical SMA materials are better-suited. The field of applications for MSMA must be found as niches where its specific characteristics can be exploited and its main drawbacks hidden. The following technical specifications were proposed to design a second version of MSMA based micro-actuator:

- The actuator must be as fast as possible (fast dynamics). This property mainly depends on the current establishment time, therefore, the coil design and the power electronics have to be improved and optimized.

- The mechanical structure must be designed to avoid backslash (for precision considerations) and friction (for efficiency considerations).

- Temperature of the device must be kept below $40{ }^{\circ} \mathrm{C}$ to avoid austenitic transformation.

- A position sensor has to be included in the final device to allow a position feedback control. The overall dimension must be kept as small as possible.

Several keynotes must be stressed for a good design with MSMA. These active materials present a strong hysteretical behaviour that must be exploited as a consequence. For example, this hysteresis should be used to keep a stable position without external supply. This allows a low energy consumption for embedded applications in space or in microrobotic fields. Authors proposed in [39] to achieve this by using a push-pull configuration as described in Figure 5. This configuration allows to replace efficiently the mechanical pre-stress device of MSMA simpler actuators. The working of this device follows these steps (see Figure 5):

- a linear simple MSMA actuator A is used to displace the positionning stage in the $+x$ direction: a magnetic field in the A airgap reorients martensite M1 of the A MSMA sample into martensite M2. This pushes the positioning stage in $+x$ direction,

- this motion stresses the linear simple actuator B and transforms its M2 martensite into M1 martensite,

- if no field is applied, the static position of the stage is kept thanks to the strong hysteresis of the stress/strain characteristic of MSMA (nevertheless, external load must be kept under a blocking stress, see Figure 3),

- the linear MSMA acturator B is used to displace the positioning stage in the $-x$ direction. The principle is the same as for the actuator A except for the direction.

A new prototype is designed according to the previous requirements. The coils are fully-optimized using both analytical and finite elements methods. The actual coil structure creates a magnetic field of $1 \mathrm{~T}$ with a current of $2 \mathrm{~A}$ 


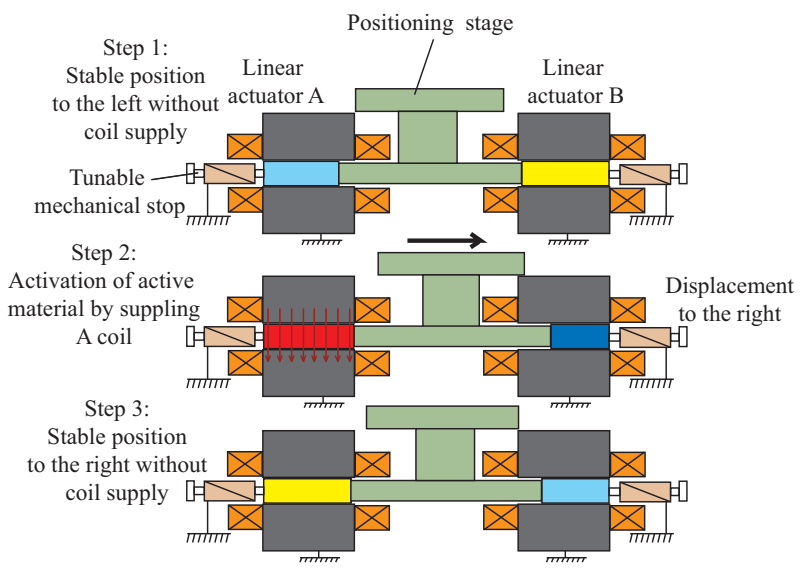

Figure 5: Description of the push-pull configuration of an actuator using two antagonist MSMA samples.

for a size of $19 \times 25 \times 25 \mathrm{~mm}^{3}$. The power electronics is designed to feed the two coils using Pulse Width Modulation (PWM) techniques and a displacement sensor is now included in the final prototype. Electronics includes a feedback control of the current, a PWM generator, a displacement feedback control and a temperature control (using micro-thermocouples). Figure 6 shows the CAD model of this device and a picture of the final prototype. The overall dimensions of this actuator are $110 \times 40 \times 30 \mathrm{~mm}^{3}$.

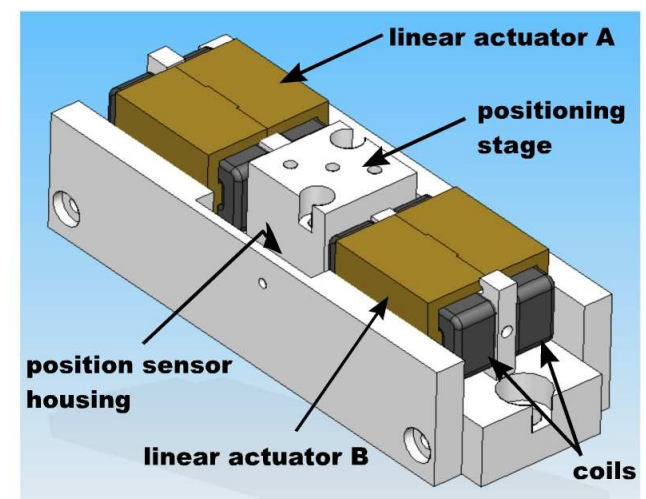

(a)

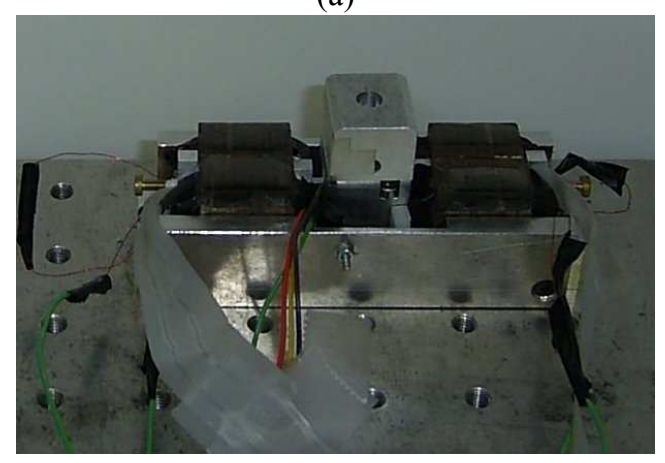

(b)

Figure 6: Push-pull actuator using two antagonist MSMA samples: (a) CAD model, (b) picture of the final prototype.
Another drawback of MSMA is its very low efficiency. It is also the case for SMA but it is amplified in this case by the magnetic actuation because of the requirement of a relatively high magnetic field density (around $1 \mathrm{~T}$ ) inside the MSMA sample to activate martensite reorientation. To reach these levels, high current are required into the coils and this leads to significant Joule effect losses. Due to heat diffusion, these losses could increase the temperature inside the MSMA and, as a consequence, cancel out the working principle of this actuator because of a martensite/austenite transformation above a temperature threshold.

To by-pass these problems, it is be possible to fed these coils by current pulses.

\subsection{Characteristics and performances}

With this new device, some measurements were conducted to extract the performances of this new MSMA-based actuator. Theses performances are summarized in the sequel:

- Displacement range: a total displacement range of approximately $1 \mathrm{~mm}$ is now possible. The range is different at high and low velocities as it was proved in [40]. These differences result mainly of different balances between energy transfer (available work) and dissipation at low and high velocities. Some results are reported in Fig. 7 for a low and a high speed motion. Without external load, the time constant of the position control is in the order of 10 milliseconds. The maximal blocking force is between 2 and $3 \mathrm{MPa}$.

- Efficiency and control by current pulses: as previously noticed, a control by current pulses is possible (see Fig. 8). This mode of working gives two mains advantages. First, the actuator keeps a position at rest without feeding: this limits the energy requirements and gives the device a high efficiency in static mode. Secondly, the actuator can be supplied by current pulses to change a position: this allows a high reduction of the mean value current and therefore a major decrease of the joule effect heating. The maximum current in the coil can be easily increased for a short time, without any austenitic transformation (only magnetic fields intensity significantly activate martensite reorientation). As this field is proportional (without saturation) to the current times the turns number of the coil, we can increase the current as well as the number of turns to increase this field. In current pulse mode, heating phenomena are lowered even if the current density is significantly increased beyond the standard levels met in classical electromagnetic devices. From the design point of view, it is also relevant to reduce the section of wires and to increase the number of turns for the coils. This allows to reduce the size of the device and to boost significantly the magnetic fields, and therefore the overall performances of the device. 
- Resolution and precision: the resolution was measured with a train of pulse (see Fig. 9), the maximal precision is about 1 micrometer and it seems, at the moment, difficult to improve this accuracy. This limit is mainly due to the complexity of the dynamic thermo-magneto-mechanical behaviour and the difficulty to achieve an accurate control (nonlinear and non-invariant system, manufacturing process defect,...). Therefore, at this time, MSMA-based actuator are hardly relevant for the high resolution actuator when the precision must be far better than micrometer. Nevertheless, for precision in the range of micrometers, MSMA is a valid alternative to other active materials.
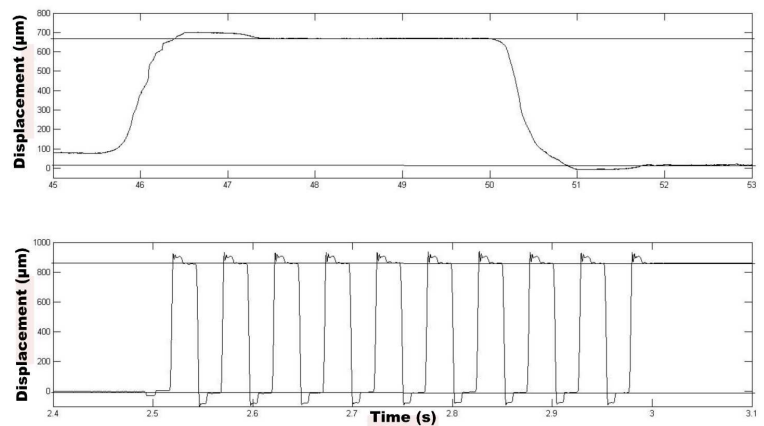

Figure 7: Displacement measurements of the MSMA-based actuator (low and high velocity modes of working).

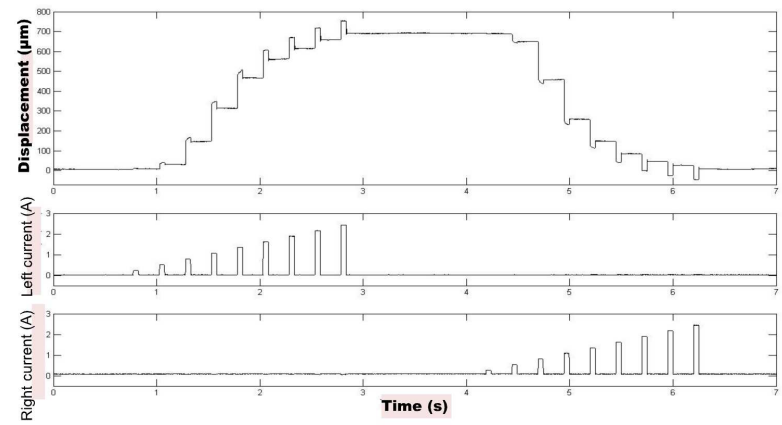

Figure 8: Current pulses control of the MSMA-based actuator.

\subsection{Control strategies}

To increase the precision and robustness, this actuator was also feedback-controlled using position and current measurements. The current sensor is a Hall-effect sensor (LTS $25 \mathrm{NP}$ from the LEM company) and the integrated position sensor is a low-cost photoelectric infrared reflexion sensor (HOA 1404-002 from the Honeywell company). This latter has a measurement range of $2 \mathrm{~mm}$ with a precision of a few micrometers depending on the analog signal processing and on the quantization of the analog to digital converter connected to the digital controller. Figure 10 represents the
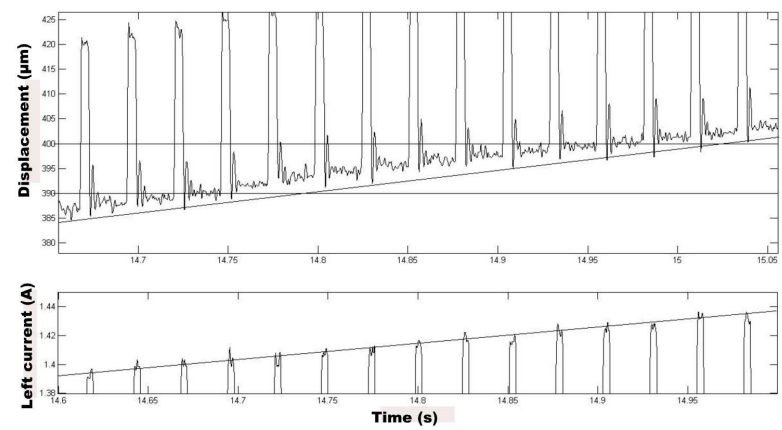

Figure 9: Resolution of the MSMA-based actuator.

block-diagram of the feedback control with a "linear" current control (without current pulse control). This feedback control is enough to give a positioning precision of a few micrometers.

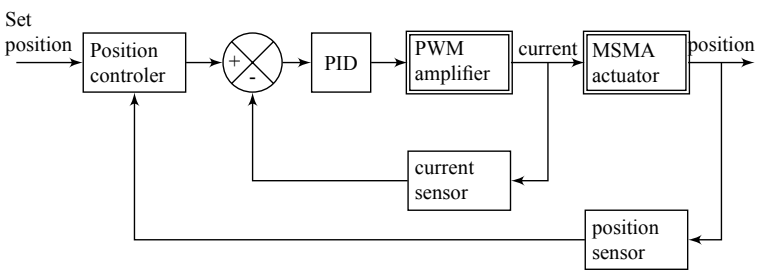

Figure 10: Block-diagram of the feedback control of MSMA-based actuator with a "linear" current control.

One of the main advantages of active materials is the possibility to use them as actuator as well as sensor. This seems also possible with MSMA materials as reported in [41]. This paper uses the change of the inductance value at low magnetic field as an indirect measurement of the stage position. This indirect measurement was also noticeable on our device and we are currently studying the self-sensing potential to remove the integrated position sensor. Nevertheless, perturbations due to the saturation of magnetic cores at high current level and to the electromagnetic radiation of the PWM power converter lead to an important signal processing treatments and to real-time problem of computation. These estimation difficulties reduce significantly the precision of the self-sensing indirect measurements in the device. Nowadays, the precision of this actuator with an integrated position sensor is far better than the precision using a self-sensing estimation principle. It will certainly be improved in the near future.

Some works are actually conducted by the authors concerning the improvement of the non-linear control of the device using port-Hamiltonian control techniques [42]. These advanced control strategies [43, 44, 45] are fullycompatible with the thermodynamics/Hamiltonian modeling presented in this paper. 


\section{Conclusion}

In this paper, the working principle of Magnetic Shape Memory Alloys was explained. An extented thermodynamics/Hamiltonian formalism was presented to model the complex non-linear magneto-thermo-mechanical behaviour of this relatively new active material. Based on this model, an application of a push-pull actuator was proposed, designed and experimented. Some measurements are reported showing a precision of a few micrometers for a displacement range of $1 \mathrm{~mm}$ with a time constant of about $10 \mathrm{mil}-$ liseconds with no load. The control of this actuator can be achieved with a pulse current feedforward control or with a current/position feedback control. Typical applications of such kind of actuators are in the micro-robotic or spatial fields with requirements between these of piezoelectric active materials and these of SMA active materials. The maximal strain of MSMA is one or two orders greater than that of piezoelectric or magnetostrictive materials and the response-time of MSMA is one or two orders smaller than that of classical SMA. For larger motions, the displacement range of this actuator can be amplified with compliant mechanical structures as is the case for actuators based on other active materials (see [46] for a amplified piezoelectric structure and [47] for an amplified magnetostrictive actuator).

Self-sensing potentials can also be pursued but currently technological improvements must be reached to replace efficiently integrated position sensor. Authors are actually focused on the control problem to improve the performance of this device using port-Hamiltonian control techniques. These ones are fully-suitable for the thermodynamics/Hamiltonian modeling presented in this paper and they can take into account the strong non-linear hysteretical behaviour of these active materials. Nevertheless, the dynamic magneto-thermo-mechanical behaviour of this material is so complex and so strongly non-linear that even today, due to a major control difficulties, the diffusion of this promising technology is dramatically reduced.

\section{Acknowledgement}

Authors would like to thank Yoann Loirat and David Guibert (Femto-ST Institute, Besançon, France) for the help during the actuator design and improvements. Authors would also thank Prof. C. Lexcellent from the Femto-ST Institute for discussions and collaborations concerning the modeling of SMA and MSMA. This work was partially supported by the project "Conception et réalisation d'actionneurs haute résolution utilisant des matériaux actifs de type alliage à mémoire de forme magnétiques" funded by the OSEOANVAR French agency (Aide aux jeunes pour l'innovation, june 2008) and by the ANR project Hamecmopsys "Hamiltonian Methods for the Control of Multidomain Distributed Parameter Systems“, Agence Nationale de la Recherche, France.

\section{References}

[1] H. Janocha, Ed., Adaptronics and Smart Structures. Basics, materials, Design, and Applications. Springer-Verlag, 1999.

[2] J. L. Pons, Emerging Actuator Technologies: A Micromechatronic Approach. John Wiley and Sons, 2005.

[3] S. Bashash and N. Jalili, "Robust adaptative control of coupled parallel piezo-flexural nanopositioning stages," IEEE/ASME Transactions on Mechatronics, vol. 14(1), pp. 11-20, February 2009.

[4] S. Polit and N. J. Dong, "Development of a highbandwidth xy nanopositioning stage for high-rate micro-/nanomanufacturing," IEEE/ASME Transactions on Mechatronics, vol. 99, pp. 1-10, June 2010.

[5] J. Y. Peng and B. Chen, "Modeling of piezoelectricdriven stick-slip actuators," IEEE/ASME Transactions on Mechatronics, vol. 16(2), pp. 394-399, April 2011.

[6] R. Merry, N. de Kleijn, N. van de Molengraft, and M. Steinbuch, "Using a walking piezo actuator to drive and control a high-precision stage," IEEE/ASME Transactions on Mechatronics, vol. 14(1), pp. 21-31, February 2009.

[7] E. du Tremolet de Lacheisserie, Magnetostriction: Theory and Applications of Magnetoelasticity. CRC Press, 1993.

[8] O. Söderberg, A. Sozinov, Y. Ge, S.-P. Hannula, and V. K. Lindroos, Handbook of Magnetic Materials, vol. 16 (Giant Magnetostrictive materials), K. H. J. Buschow, Ed. Elsevier, 2005.

[9] D. Davino, A. Giustiniani, and C. Visone, "Design and test of a stress-dependant compensator for magnetostrictive actuators," IEEE Transactions on Magnetics, vol. 46(2), pp. 646-649, January 2010.

[10] Y. Sato and K. Shinohara, "Power-saving drive in 2position control of giant-magnetostrictive actuator," IEEE Transactions on Magnetics, vol. 45(10), pp. 4554-4557, September 2009.

[11] W.-J. Kim and A. Sadighi, "A novel low-power linear magnetostrictive actuator with local three-phase excitation," IEEE/ASME Transactions on Mechatronics, vol. 15(2), pp. 299-307, March 2010.

[12] S. J. Murray, M. Marioni, S. M. Allen, R. C. O'Handley, and T. A. Lograsso, " 6 percent magneticfield-induced strain by twin-boundary motion in ferromagnetic Ni-Mn-Ga," Applied Physical Letters, vol. 77, pp. 886-8, 2000. 
[13] K. Ullakko, J. K. Huang, C. Kantner, R. C. O'Handley, and V. V. Kokorin, "Large magnetic-fieldinduced strains in Ni2MnGa single crystals," in Applied Physics Letters, vol. 69, no. 13, 1996, pp. 19661968.

[14] R. D. James and M. Wuttig, "Magnetostriction of martensite," in Philosophical Magazine A, vol. 77, no. 5, 1998, pp. $1273-1299$.

[15] O. Söderberg, Y. Ge, A. Sozinov, S.-P. Hannula, and V. K. Lindroos, "Recent breakthrough development of the magnetic shape memory effect in Ni-Mn-Ga alloys," IOP Smart Materials and Structures, vol. 14, pp. 223-335, 2005.

[16] J. Pons, E. Cesari, C. Seguì, F. Masdeu, and R. Santamarta, "Ferromagnetic shape memory alloys: Alternatives to Ni-Mn-Ga," Materials Science and Engineering A, vol. 481-482, pp. 57-65, 2008.

[17] M. Kohl, D. Brugger, and B. Krevet, "Ferromagnetic shape memory actuator for large 2 d optical scanning," in 19th IEEE International Conference on Micro Electro Mechanical Systems, Istanbul, Turkey, 2006.

[18] M. Kohl, D. Brugger, M. Ohtsuka, and B. Krevet, "A ferromagnetic shape memory actuator designed for large 2d optical scanning," Sensors and Actuators A, vol. 135, pp. 92-98, 2007.

[19] R. Tickle and R. D. James, "Magnetic and magnetomechanical properties of $\mathrm{Ni}_{2} \mathrm{MnGa}$," Journal of Magnetism and Magnetic Materials, vol. 195, pp. 627-638, juin 1999.

[20] A. DeSimone and R. D. James, "A constrained theory of magnetoelasticity," Journal of the Mechanics and Physics of Solids, vol. 50, no. 2, pp. 283-320, 2002.

[21] P. Müllner, V. Chernenko, and G. Kostorz, "A microscopic approach to the magnetic-field-induced deformation of martensite (magnetoplasticity)," Journal of Magnetism and Magnetic Materials, vol. 267, pp. 325-334, 2003.

[22] R. Ahluwalia, T. Lookman, and A. Saxena, "Dynamic strain loading of cubic to tetragonal martensites," Acta materialia, vol. 54, pp. 2109-2120, 2006.

[23] O. Heczko, "Determination of ordinary magnetostriction in Ni-Mn-Ga magnetic shape memory alloy," Journal of Magnetism and Magnetic Materials, vol. 290-291, pp. 846-849, 2005.

[24] B. Kiefer and D. C. Lagoudas, "Magnetic fieldinduced martensitic variant reorientation in magnetic shape memory alloys," Philosophical Magazine Special Issue: Recent Advances in Theoretical Mechanics, in Honor of SES 2003 A.C. Eringen Medalist G.A. Maugin, vol. 85(33-35), pp. 4289-4329, 2005.
[25] R. N. Couch and I. Chopra, "A quasi-static model for NiMnGa magnetic shape memory alloy," Smart Materials and Structures, vol. 16, pp. S11-S21, 2007.

[26] R. C. Smith, M. J. Dapino, T. R. Braun, and A. P. Mortensen, "A homogenized energy framework for ferromagnetic hysteresis," IEEE Transactions on Magnetics, vol. 42(7), pp. 1747-1769, 2006.

[27] N. N. Sarawate and M. J. Dapino, "A continuum thermodynamics model for the sensing effect in ferromagnetic shape memory Ni-Mn-Ga," Journal of applied physics, vol. 101, pp. 123 522-1-123 522-11, 2007.

[28] J. Y. Gauthier, C. Lexcellent, A. Hubert, J. Abadie, and N. Chaillet, "Modeling rearrangement process of martensite platelets in a magnetic shape memory alloy $\mathrm{Ni}_{2} \mathrm{MnGa}$ single crystal under magnetic field and (or) stress action," Journal of Intelligent Material Systems and Structures, vol. 18, no. 3, pp. 289-299, 2007.

[29] — - "Magneto-thermo-mechanical modeling of a magnetic shape memory alloy Ni-Mn-Ga single crystal," Annals of Solid and Structural Mechanics, vol. 2, no. 1, pp. 19-31, 2011.

[30] H. B. Callen, Thermodynamics and an introduction to thermostatistics, $2^{\text {nde }}$ edition. John Wiley and Sons, 1985.

[31] J. Y. Gauthier, A. Hubert, J. Abadie, N. Chaillet, and C. Lexcellent, "Nonlinear hamiltonian modelling of magnetic shape memory alloy based actuators," Sensors \& Actuators A, vol. 141, pp. 536-547, 2008.

[32] C. Lanczos, The Variational Principe of Mechanics, Fourth edition. Dovers Publications, Reprint of 1970 University of Toronto Press, 1986.

[33] H. Goldstein, Classical Mechanics, second edition. Addison Wesley, 1980.

[34] I. Suorsa, J. Tellinen, E. Pagounis, I. Aaltio, and K. Ullakko, "Applications of magnetic shape memory actuators," in 8th international conference ACTUATOR 2002, Bremen (Germany), 2002.

[35] I. Suorsa, J. Tellinen, I. Aaltio, E. Pagounis, and K. Ullakko, "Design of active element for MSMactuator," in ACTUATOR 2004 / 9th International Conference on New Actuators, Bremen (Germany), juin 2004.

[36] I. Suorsa, E. Pagounis, and K. Ullakko, "Magnetic shape memory actuator performance," Journal of Magnetism and Magnetic Materials, vol. 272-276, pp. 2029-2030, 2004.

[37] F.-X. Wang, W.-J. Li, Q.-X. Zhang, X.-J. Wu, and C. Ge, "Design and control of linear actuators made by magnetically controlled shape memory alloy," in IEEE International Conference on Mechatronics, July 10-12 2005, pp. 583-586. 
[38] Q.-X. Zhang and F.-X. Wang, "A novel linear actuator and its controlling system," in IEEE International Conference on Automation and Logistics, August 1821 2007, pp. 979-984.

[39] J. Y. Gauthier, A. Hubert, J. Abadie, C. Lexcellent, and N. Chaillet, "Multistable actuator based on magnetic shape memory alloy," in ACTUATOR 2006, 10th International Conference on New Actuators, Bremen, Germany, 2006, pp. 787-790.

[40] — - "Original hybrid control for robotic structures using magnetic shape memory alloys actuators," in IEEE/RSJ International Conference on Intelligent Robots and Systems, 2007, pp. 787-790.

[41] I. Suorsa, E. Pagounis, and K. Ullakko, "Position dependent inductance based on magnetic shape memory materials," Sensors and Actuators A, vol. 121, pp. 136-141, 2005.

[42] N. Calchand, A. Hubert, Y. Le Gorrec, and B. Mashke, "From canonical hamiltonian to port-hamiltonian modeling. Application to magnetic shape memory alloys actuators," in ASME 2011 Dynamic Systems and Control Conference (DSCC'2011), Airlington, Virginia, USA, 31 oct.-01 nov. 2011.

[43] A. J. van der Schaft, $L_{2}$-Gain and Passivity Techniques in Nonlinear Control. Springer-Verlag, 2000.

[44] A. Astolfi and A. J. van der Schaft, Eds., Lagrangian and Hamiltonian Methods for Nonlinear Control, ser. Special Issue of European Journal of Control, Volume 10, Number 5. Hermes, 2004.

[45] B. Brogliato, R. Lozano, B. Maschke, and O. Egeland, Dissipative Systems Analysis and Control. Theory and applications, $2^{\text {nde }}$ edition. Springer-Verlag, 2007.

[46] M. Grossard, M. Boukallel, N. Chaillet, and C. Rotinat-Libersa, "Modeling and robust control strategy for a control-optimized piezoelectric microgripper," IEEE/ASME Transactions on Mechatronics, vol. 99, pp. 1-10, June 2010.

[47] F. Claeyssen, N. Lhermet, F. Barillot, and R. L. Letty, "Giant dynamic strains in magnetostrictive actuators and transducers," in ISAGMM, Guiyang, China, October 9-12 2006. 